FERMILAB-TM-2365

April 18, 2007

\title{
The DØ Experiment's Integrated Luminosity for Tevatron Run IIa
}

T. Andeen ${ }^{(\mathrm{d})}$, B. C. K. Casey ${ }^{(\mathrm{a})}$, K. DeVaughan ${ }^{(\mathrm{e})}$, Y. Enari ${ }^{(\mathrm{a})}$, E. Gallas ${ }^{(\mathrm{b})}$, D. Krop ${ }^{(\mathrm{c})}$,

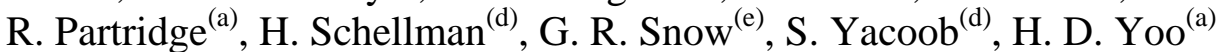

${ }^{(a)}$ Brown University, ${ }^{(b)}$ Fermi National Accelerator Laboratory, ${ }^{(c)}$ Indiana University,

${ }^{(d)}$ Northwestern University, ${ }^{(e)}$ University of Nebraska

\section{Introduction}

An essential ingredient in all cross section measurements is the luminosity used to normalize the data sample. In this note, we present the final assessment of the integrated luminosity recorded by the DØ experiment during Tevatron Run IIa. The luminosity measurement is derived from hit rates from the products of inelastic proton-antiproton collisions registered in two arrays of scintillation counters called the luminosity monitor (LM) detectors. Measured LM rates are converted to absolute luminosity using a normalization procedure that is based on previously measured inelastic cross sections and the geometric acceptance and efficiency of the LM detectors for registering inelastic events.

During Run IIa, the LM detector performance was improved by a sequence of upgrades to the electronic readout system and other factors summarized in this note. The effects of these changes on the reported luminosity were tracked carefully during the run. Due to the changes, we partition the run into periods for which different conversions from measured LM rates to absolute luminosity apply. The primary upgrade to the readout system late in Run IIa facilitated a reevaluation of the overall normalization of the luminosity measurement for the full data sample.

In this note, we first review the luminosity measurement technique employed by DØ. We then summarize the changes to the LM system during Run IIa and the corresponding normalization adjustments. The effect of the adjustments is to increase DØ's assessment of its recorded integrated luminosity compared to what was initially reported during Run IIa. The overall increase is $13.4 \%$ for data collected between April 20, 2002 (the beginning of Run IIa data used for physics analysis) and February 22, 2006 (the end of Run IIa).

\section{The DØ Luminosity Measurement Technique}

The luminosity, $L$, is determined by measuring the counting rate of inelastic protonantiproton collisions recorded by the DØ luminosity monitor system [1]:

$$
L=\frac{1}{\sigma_{e f f}} \frac{d N}{d t},
$$


where $\sigma_{\text {eff }}$ is the effective inelastic cross section seen by the LM. The effective cross section is derived from the total inelastic cross section, $\sigma_{\text {inelastic }}(1.96 \mathrm{TeV})=60.7 \pm 2.4$ $\mathrm{mb}$ [2], used by both the DØ and CDF collaborations for Run II luminosity determinations. The cross section $\sigma_{\text {eff }}$ differs from $\sigma_{\text {inelastic }}$ by the LM system geometric acceptance and efficiency for registering inelastic events. Both the acceptance and efficiency are determined from Monte Carlo simulated inelastic events run through a detailed simulation of the LM detector and response. The LM consists of two arrays of 24 plastic scintillators radiating outward from the beam pipe located at $z= \pm 140 \mathrm{~cm}$ where the positive $z$ direction is the proton beam direction. The arrays, shown in Fig. 1, cover the pseudo-rapidity region $2.7<|\eta|<4.4$.
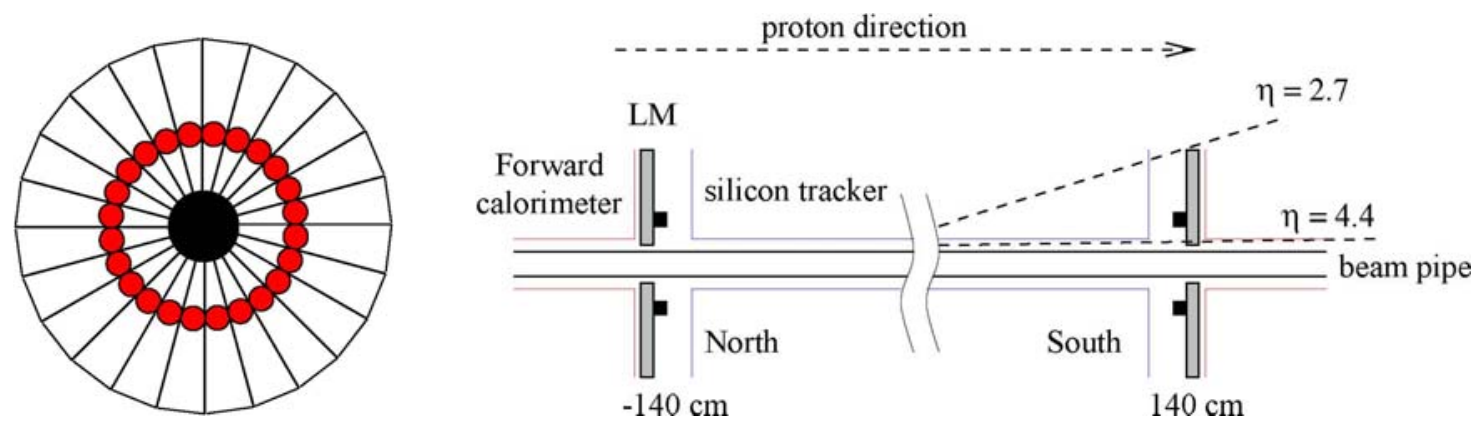

Figure 1. The Luminosity Monitor layout. The $r$ - $\varphi$ view is on the left. The $r$-z view of the two arrays is shown on the right.

In practice, the luminosity is calculated by inverting the expression

$$
P(0)=e^{-\sigma_{\text {eff }} L / f} \times\left(2 e^{\left(-\sigma_{S S} / 2 f\right) L}-e^{-\sigma_{s S} L / f}\right),
$$

where $P(0)$ is the probability that a bunch crossing does not produce a coincidence of intime hits recorded in each of the two LM arrays, $\sigma_{S S}$ ( $S S \equiv$ single-sided) is the effective cross section for only one of the arrays to fire, and $f$ is the crossing frequency for a given beam bunch. This approach is referred to as the counting zeros technique. During datataking, $P(0)$ is measured for each of the 36 separate bunch crossings. Each $P(0)$ is determined by counting events over a time period of about one minute, chosen long enough that the statistical uncertainty on $P(0)$ is much less than $1 \%$, but short enough that the instantaneous luminosity changes negligibly. The luminosity-dependent term in parentheses on the right side of equation (2) is due to the presence of multiple interactions per bunch crossing. This term accounts for beam crossings that have no proton-antiproton interactions that produce hits in both luminosity arrays, but have two or more interactions that each fire a single array and jointly produce hits in both arrays.

\section{Evolution of the LM system during Run IIa}

Run IIa began with the LM scintillator signals processed with a NIM-based electronics system that had been used for the Run I luminosity monitors called the Level-0 detectors. Run IIa data collection used for DØ physics analysis began on April 20, 2002. The NIM 
system was used to report the luminosity until October 20, 2005. On this date, the reporting of luminosity was switched to a custom-built VME-based electronics system [3] that is intended to be used until the end of Run II.

The NIM and VME readout systems employed the same basic technique for counting inelastic events and relied on coincident hits in each of the two 24-scintillator arrays. In the NIM system, the 24 analog photomultiplier (PMT) signals from each LM detector side were summed. The summed signal for each side was then discriminated and required to have timing consistent with a proton-antiproton interaction. The NIM system did not provide information on the number of scintillators firing, nor on their individual pulse heights or hit times. The VME system, which discriminates the 48 individual analog signals, provides timing and pulse height information for each scintillator. These electronics have a much larger dynamic range than the previous system. By raising the PMT voltages and discriminating individual PMT signals, rather than discriminating the analog sum of 24 such signals, we are able to achieve approximately $100 \%$ efficiency for inelastic events with particles within the geometric acceptance of the LM scintillators. This is demonstrated by the plateau curve in Fig. 2, indicating that a further increase of $50 \%$ in the phototube gain would only increase the reported luminosity by $0.3 \%$.

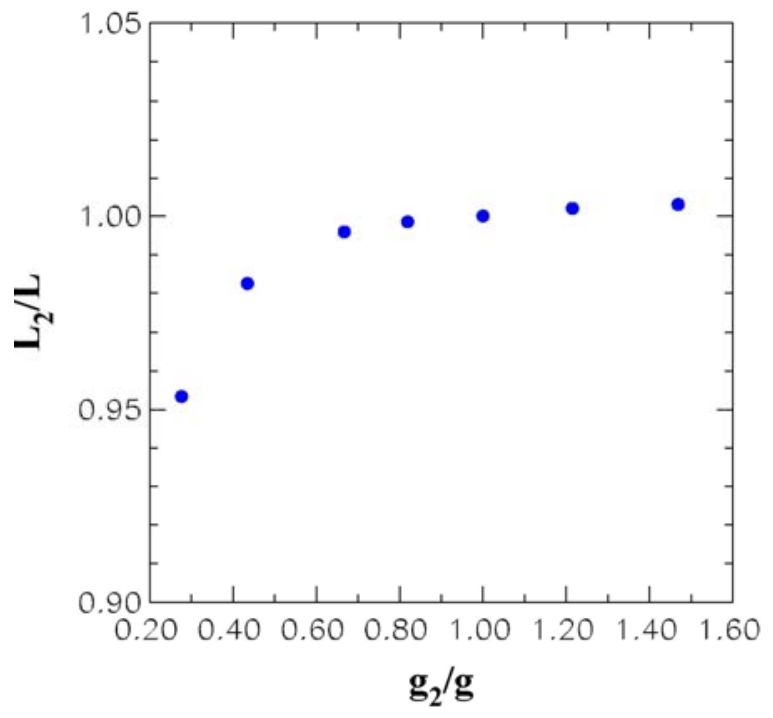

Figure 2. The relative change in reported luminosity versus a relative change in the gain of the LM detector photomultiplier tubes. A 50\% increase in the gain changes the reported luminosity by less than $0.3 \%$, indicating that the operating point $\left(\mathrm{g}_{2} / \mathrm{g}=1.0\right.$ ) yields very close to $100 \%$ efficiency for counting inelastic collisions with particles in the LM detector acceptance.

While the NIM system was in use, three changes affected the performance of the LM system. The first change was external to the LM system. From the beginning of Run IIa until the Fall 2004 shutdown (start date August 23, 2004), the central solenoid magnet current was 4750 A. From the end of the shutdown on December 7, 2004, through the end of Run IIa, the solenoid magnet operated with a reduced current of 4550 A. The LM detectors sit in the diverging magnetic field emerging from the solenoid. The reduction in 
magnetic field strength increased the LM phototube gains, which resulted in a small increase in the LM efficiency for registering inelastic collisions. This calls for a corresponding adjustment to the luminosity normalization for data collected before the Fall 2004 shutdown relative to the end of Run IIa. To quantify the effect, the reported luminosity was measured as a function of changing magnetic field current over a short period during which the true luminosity was approximately constant. The measurement revealed that the LM system was $(2.6 \pm 0.6) \%$ less efficient for registering inelastic collisions at the higher current value employed before the Fall 2004 shutdown. The normalization adjustment is referred to as the magnet correction in Section $\mathrm{V}$ below.

The second change was a modification to the NIM electronics system to remove baseline shifts that were observed at the input to the discriminator for each LM detector side. As described above, the 24 analog signals from each LM detector array were summed in commercial NIM modules whose output can carry a DC offset. To minimize the effect of offset drift, the summed output signals were AC coupled before the NIM discriminators. This led to a small luminosity-dependent inefficiency in the LM response to inelastic events. The problem was remedied in two sequential steps which both minimized the effect of the baseline shifts. The first step occurred on December 19, 2004, when the RC time constant for the AC coupling was shortened to better restore the baseline before the next beam crossing. Then in March 2005, a custom-made NIM module was introduced that sampled the baseline during abort gaps as a reference and performed active baseline restoration using a feedback circuit. To quantify the effect, the reported luminosity was measured with and without the baseline restoration at several values of instantaneous luminosity. A fit to the data points yields a luminosity-dependent correction function that can be used to adjust the measured luminosity before the baseline restoration was implemented. The function reveals that the LM system was between $0 \%$ and $3 \%$ less efficient for registering inelastic collisions before the baseline restoration. The normalization adjustment is referred to as the baseline correction in Section V below.

The third change was a modification to the NIM electronics system to correct for dead time that prevented the LM detectors from identifying the presence of an inelastic collision in particular beam crossings. The source of the dead time was out-of-time hits thought to be due to slow neutrons and/or detector activation from energy depositions in previous bunch crossings. (Dead time due to beam halo particles, which cause out-oftime hits at a predictable time, had already been accounted for in the system.) An electronic solution to minimize dead time in the system was implemented on March 17, 2005. New electronics to monitor dead time and make a corresponding online correction were also installed. The effect of the dead time correction on the reported luminosity is quantified, as above, with a luminosity-dependent correction function derived from measurements made with and without the electronic solution in the readout chain. Before the dead time solution was implemented, the LM system was between 0\% (at zero instantaneous luminosity) and 20\% (at $120 \times 10^{30} \mathrm{~cm}^{-2} \mathrm{~s}^{-1}$ ) less efficient for registering inelastic collisions. The average correction is only a few percent since little integrated luminosity was recorded at instantaneous values approaching $120 \times 10^{30} \mathrm{~cm}^{-2} \mathrm{~s}^{-1}$ before the dead time solution was implemented. The normalization adjustment is referred to as the dead time correction in Section $\mathrm{V}$ below. 
In addition to the above changes, the LM scintillators suffered mild radiation damage during their exposure to over $1 \mathrm{fb}^{-1}$ integrated luminosity during Run IIa. After dismounting the LM detectors at the end of Run IIa, measurements [4] revealed that the light yield from the exposed scintillators was reduced by $10-15 \%$ compared to new scintillators. Since the light yield affects the efficiency of the LM system to register inelastic collisions, the radiation damage calls for a correction to the luminosity normalization that is a function of the integrated exposure of the scintillators to the particle flux during Run IIa. The effect on the normalization is quantified by appropriately adjusting the light yield profiles used in the Monte Carlo simulation of the scintillator response. This leads to the conclusion that the LM system was about $2.5 \%$ more efficient for registering inelastic collisions at the beginning of Run IIa relative to the end. The normalization adjustment is referred to as the radiation damage correction in Section $\mathrm{V}$ below. We note for completeness that the LM scintillators were replaced before the start of Run IIb.

\section{Determination of $\sigma_{\text {eff }}$ and $\sigma_{\text {sS }}$ for Run IIa}

During Run IIa, two sets of values for $\sigma_{\text {eff }}$ and $\sigma_{S S}$ were used in the calculation of the luminosity that was reported internally to the experiment and externally to the laboratory. The values of $\sigma_{\text {eff }}=46.0 \mathrm{mb}$ and $\sigma_{S S}=6.3 \mathrm{mb}$ were determined [5] and applied during the time the NIM electronics system reported the luminosity. These values stem from a technique for determining the LM detector efficiency that relied on the energy deposition in the calorimeter cells located behind the LM scintillators. The LM detector acceptance was determined using Monte Carlo. When the VME-based electronics system began reporting the luminosity, the increase in the LM efficiency would have led to a sudden increase in the reported luminosity. The $\sigma_{\text {eff }}$ value was adjusted to an interim value of $54.0 \mathrm{mb}$ so that the luminosity reported by the VME system would match the luminosity reported by the NIM system until a final value appropriate to the VME system could be determined. The $\sigma_{S S}$ value was not changed at this time. The latter values $\left(\sigma_{\text {eff }}=54.0 \mathrm{mb}\right.$ and $\sigma_{S S}=6.3 \mathrm{mb}$ ) were used through the end of Run IIa. Subsequently, information from the VME system allowed us to determine improved values for $\sigma_{\text {eff }}$ and $\sigma_{S S}$, as discussed below, which were implemented on September 29, 2006.

The matching mentioned in the preceding paragraph was performed at an instantaneous luminosity of about $20 \times 10^{30} \mathrm{~cm}^{-2} \mathrm{~s}^{-1}$. A small luminosity-dependent adjustment to the NIM to VME matching was quantified by comparing the reported luminosity from the NIM and VME systems at several values of instantaneous luminosity. A fit to the data points leads to a correction function that varies between $-1 \%$ and $+2 \%$ and is applied to all data taken before the NIM to VME switch. The normalization adjustment is referred to as the NIM to VME correction in Section V below.

Data taken with the VME system was subsequently used to make an improved determination of the normalization constants. The new pulse height and timing information allowed us to perform detailed calibration of the LM detectors on a channel by channel basis and make measurements of the single channel response of the system. This information, in turn, allowed us to produce a more accurate simulation of the LM 
detectors for determining the acceptance and efficiency for registering inelastic collisions.

With the single counter response properly simulated and the detector counting rate plateaued for inelastic collisions (see Fig. 2), the remaining ingredient is the fraction of inelastic collisions with particles in the acceptance of either array but not both. These events are predominantly caused by diffractive collisions where either the proton or antiproton remains intact in the inelastic collision. To account for this, we generate separate samples of diffractive and non-diffractive Monte Carlo events. The appropriate value of the non-diffractive fraction $\left(f_{N D}\right)$ is determined by varying the weight of the two sets of Monte Carlo events until the best agreement with a chosen zero-bias data sample is achieved. Specifically, we compare the measured multiplicity distributions of LM counters above threshold with the combined diffractive and non-diffractive Monte Carlo distributions constructed with different values of $f_{N D}$. For each value of $f_{N D}$, we calculate a $\chi^{2}$ between data and Monte Carlo in two multiplicity bins (zero and greater than zero) and add the $\chi^{2}$ values for the north and south LM arrays. The final $f_{N D}$ value results from minimizing the summed $\chi^{2}$.
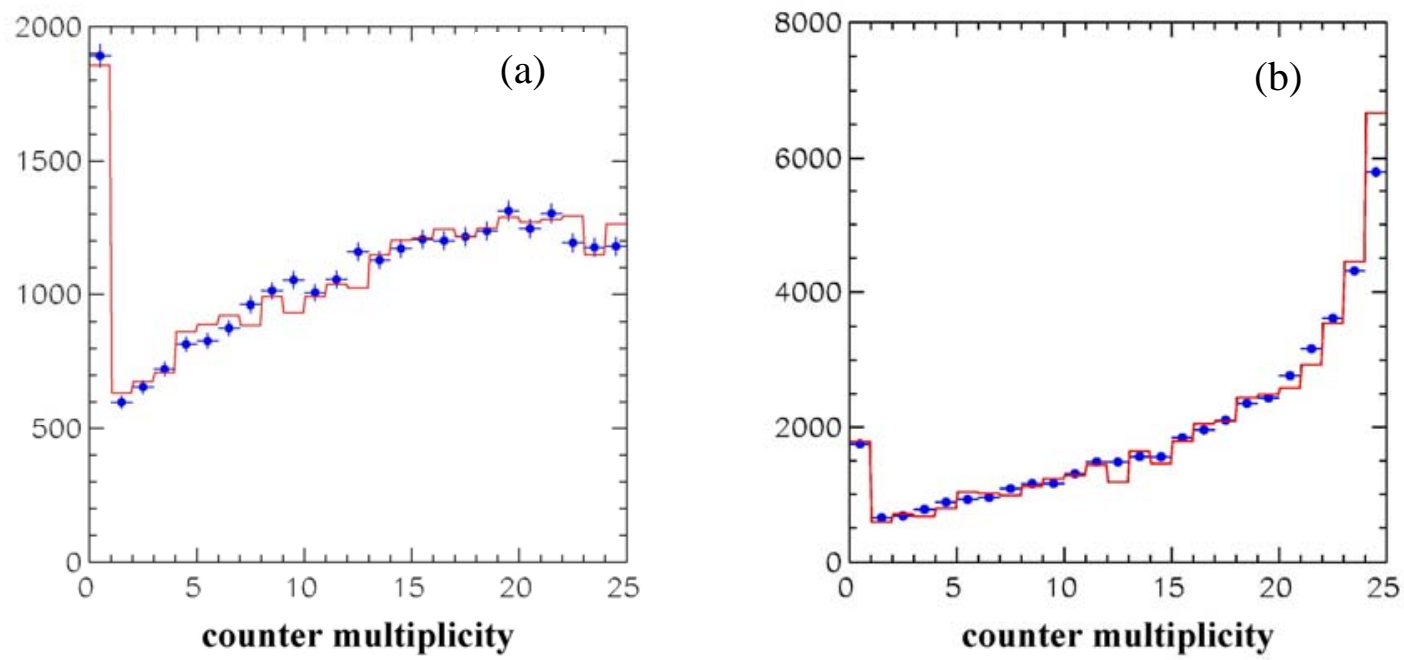

Figure 3. The multiplicity of counters above threshold in the LM detectors. The sum of the north and south LM distributions are plotted. In each plot, the points with error bars are zero-bias data and the solid histogram is derived from the inelastic Monte Carlo used to determine the acceptance for inelastic collisions. Plot (a) corresponds to an instantaneous luminosity of $13 \times 10^{30} \mathrm{~cm}^{-2} \mathrm{~s}^{-1}$ and plot (b) to an instantaneous luminosity of $45 \times 10^{30} \mathrm{~cm}^{-2} \mathrm{~s}^{-1}$.

Examples of such data and Monte Carlo multiplicity distributions are shown in Fig. 3. Fig. 3(a) results from the $\chi^{2}$ fitting procedure to determine $f_{N D}$ which is described above. The data distribution corresponds to an instantaneous luminosity of $13 \times 10^{30} \mathrm{~cm}^{-2} \mathrm{~s}^{-1}$ and the number of interactions per bunch crossing at this luminosity was appropriately modeled in the Monte Carlo. We note that the Monte Carlo reproduces the shape of the data distribution over the full multiplicity range even though the fitting procedure relied 
on only the zero bin and the sum of bins with multiplicity greater than zero. Fig. 3(b) shows the data and Monte Carlo multiplicity distributions for a higher instantaneous luminosity, $45 \times 10^{30} \mathrm{~cm}^{-2} \mathrm{~s}^{-1}$. In this plot, the Monte Carlo used the $f_{N D}$ value determined from Fig. 3(a) and a modeling of the number of interactions that corresponds to this luminosity. The Monte Carlo agrees with the data's zero bin and reasonably reproduces the shape of the nonzero data bins with no further adjustment to the Monte Carlo.

The new simulation leads to final effective cross sections of $\sigma_{\text {eff }}=48.0 \mathrm{mb}$ and $\sigma_{S S}=9.4$ mb. Comparing $\sigma_{\text {eff }}=48.0 \mathrm{mb}$ to the value $54.0 \mathrm{mb}$ above reveals that the reported luminosity was underestimated by $12.5 \%$ at the end of Run IIa for low instantaneous luminosities for which the multiple interaction term in equation (2) is negligible. The degree of underestimation is luminosity dependent due to the multiple interaction term. Replacing the previous $\sigma_{S S}=6.3 \mathrm{mb}$ above with the final $\sigma_{S S}=9.4$ indicates, for example, that the reported luminosity was underestimated by about $10 \%$ at $120 \times 10^{30} \mathrm{~cm}^{-2} \mathrm{~s}^{-1}$.

Several cross checks have been performed to demonstrate the robustness of the final effective cross sections. These include large variations in the Monte Carlo model used to simulate inelastic events, in the material description in front of the LM detector in the Monte Carlo, and in the multiple interaction model used to compare the Monte Carlo distributions with data taken at luminosities with approximately 0.4 interactions per event. The weighting of the diffractive and non-diffractive Monte Carlo samples is also varied within a range that gives acceptable agreement between the data and Monte Carlo multiplicity distributions. From these studies we derive an uncertainty of $5.4 \%$ on the effective cross sections which includes the $4.0 \%$ uncertainty on the total inelastic cross section, $\sigma_{\text {inelastic }}(1.96 \mathrm{TeV})$ [2], used by the CDF and $\mathrm{D} \varnothing$ experiments for luminosity determinations.

The re-determination of $\sigma_{\text {eff }}$ and $\sigma_{S S}$ revealed weaknesses in the previous determination of these normalization constants. We understand now that the LM efficiency was previously overestimated due to the method used. In addition, the counter-by-counter information available from the VME system allowed a direct determination of the diffractive to nondiffraction cross section ratio. Previously, we had relied on a value for the ratio determined by other experiments [6,7,8]. The more precisely tuned Monte Carlo also revealed that the LM acceptances were previously overestimated. For each of these ingredients, the few percent differences between the previously determined and the presently understood values are within the previously quoted systematic uncertainty. However, the differences are all in the same direction and lead to an accumulated correction larger than the previously quoted systematic uncertainty. These and other small effects quantified by the new simulation account for the previous underestimation of the luminosity at the end of Run IIa.

The effects of the final normalization constants and the other corrections described in Sections III and IV on the assessed integrated luminosity for Run IIa are quantified in Sections V and VI. 


\section{Back propagation of the final normalization constants and other adjustments}

The changes to the LM system described in the above sections partition Run IIa into 5 data-taking periods, called A through E, for which separate adjustments to the luminosity normalization are required. Table 1 lists the 5 periods, the date ranges that define them, and the normalization adjustments that are applied. Note that an adjustment listed for a given period is applied to that period and all prior periods in a multiplicative fashion. As an example, period B is affected by all the corrections except the magnet correction. This correction procedure is therefore referred to as the back propagation of the normalization adjustments. Although the effect of the scintillator radiation damage is assumed to have accumulated in a continuous fashion in proportion to the integrated luminosity exposure during Run IIa, we apply a different constant adjustment to the integrated luminosity for each of the periods for simplicity. Since the radiation damage corrections are small (1$2 \%$ ), the difference between applying per-period constant adjustments and continuous adjustments is negligible.

\begin{tabular}{|l|l|l|}
\hline Period & Date range & Corrections applied \\
\hline A & $4 / 20 / 2002$ to $8 / 23 / 2004$ & Magnet correction \\
\hline B & $12 / 7 / 2004$ to $12 / 19 / 2004$ & Baseline correction \\
\hline C & $12 / 19 / 2004$ to $3 / 17 / 2005$ & Dead time correction \\
\hline D & $3 / 17 / 2005$ to $10 / 20 / 2005$ & NIM to VME and radiation damage corrections \\
\hline E & $10 / 20 / 2005$ to $2 / 22 / 2006$ & Final $\sigma_{\text {eff }}$ and $\sigma_{\text {SS }}$ determination \\
\hline
\end{tabular}

Table 1. The 5 data taking periods in Run IIa that require separate luminosity normalization adjustments.

\section{Adjusted Run IIa recorded luminosity for the experiment's highest $E_{T}$ jet trigger}

All of the corrections listed in Table 1, apart from the magnet correction, are described by luminosity-dependent, analytic functions derived from measurements. The magnet correction is a constant offset. Hence, to assess the net effect of the corrections on the integrated luminosity for a given period, the accumulated correction functions must be convoluted with the instantaneous luminosity profiles (integrated luminosity recorded in bins of instantaneous luminosity) for that period. As an example, Fig. 4 shows the uncorrected and corrected luminosity profiles for period A.

We note that the recorded luminosity profiles and values referred to in this section correspond to the luminosity exposure of the experiment's highest $E_{T}$ jet trigger. This trigger, which requires at least one jet with $E_{T}>125 \mathrm{GeV}$, is never prescaled and is present in every data-taking run used for physics analysis. Not included is the luminosity recorded in special runs used for detector diagnostics and calibration which amounts to less than 3\% of the recorded luminosity used for physics analysis. 


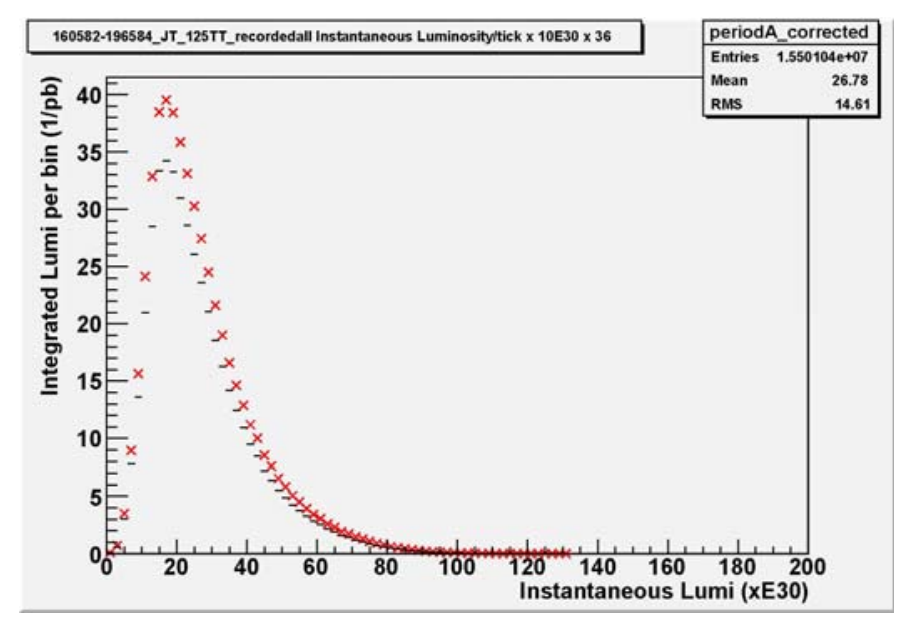

Figure 4. The luminosity profile for period $A$ in bins of width $2 \times 10^{30} \mathrm{~cm}^{-2} \mathrm{~s}^{-1}$ recorded with the highest $E_{T}$ jet trigger described in the text above. The black points (dashes) are

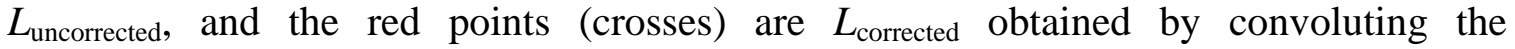
accumulated correction functions for this period with the uncorrected profile.

The net effect on the integrated luminosity for a given period is obtained by integrating the corrected profile distribution. Table 2 summarizes the adjustments to the assessed recorded integrated luminosity for the 5 periods in Run IIa. Again, the values in Table 2 correspond to the experiment's highest $E_{T}$ jet trigger. The last row of the table indicates that the recorded luminosity for Run IIa was underestimated by $13.4 \%$. To ensure internal consistency, the adjustments to the Run IIa assessed luminosity have been cross checked with measured rates of physics processes that are independent of the LM system. The adjusted luminosity values have been incorporated into the offline software tools employed by $\mathrm{D} \varnothing$ collaborators to obtain the integrated luminosity for data samples used in physics analyses.

\begin{tabular}{|l|l|l|l|}
\hline Period & Uncorrected L (pb & $\mathbf{- 1})$ & Corrected L (pb \\
\hline A & ) & \% Increase \\
\hline B & 451 & 525 & 16.5 \\
\hline C & 6.8 & 7.8 & 14.6 \\
\hline D & 125 & 142 & 13.8 \\
\hline E & 394 & 436 & 10.6 \\
\hline Total & 183 & 204 & 11.5 \\
\hline
\end{tabular}

Table 2. Summary of the corrections to the integrated luminosity in Run IIa. The integrated luminosity values correspond to the exposure of the experiment's highest $E_{T}$ jet trigger as described in the text.

The uncertainty the $\mathrm{D} \varnothing$ experiment assigns to the measured luminosity for Run IIa is $6.1 \%$. This is the uncertainty estimated for period A whose adjusted integrated luminosity is affected by all the normalization corrections and amounts to about half of the total Run IIa luminosity. The dominant contribution is $5.4 \%$, the estimated uncertainty in the determination of the final value of $\sigma_{\text {eff }}=48.0 \mathrm{mb}$. This uncertainty has approximately 
equal contributions from uncertainties in $\sigma_{\text {inelastic }}(1.96 \mathrm{TeV})$ described in [2] and uncertainties in the determination of the acceptance and efficiency of the LM detectors for registering inelastic collisions. The final $6.1 \%$ uncertainty results from the quadrature addition of the uncertainties associated with the back propagation procedure with the above 5.4\%. These include fit uncertainties in the analytic correction functions derived from measurements and an estimate of the uncertainty associated with the effect of scintillator radiation damage.

\section{Conclusions}

We have presented the final assessment of the integrated luminosity recorded by the DØ experiment during Run IIa. The recorded luminosity corresponding to the experiment's highest $E_{T}$ jet trigger is $(1315 \pm 80) \mathrm{pb}^{-1}$ (i.e. relative uncertainty of $\left.6.1 \%\right)$. When the luminosity recorded in special runs is included, the adjusted integrated recorded luminosity is $1350 \mathrm{pb}^{-1}$. The final assessment of the integrated luminosity is $13.4 \%$ greater than that reported at the end of Run IIa.

\section{References}

[1] C-C. Miao, FERMILAB-Conf-98/395-E (1998); V.M. Abazov et al., Nucl. Instr. and Methods A565, 463 (2006), FERMILAB-Pub-05/341-E (2005).

[2] S. Klimenko, J. Konigsberg, T.M. Liss, FERMILAB-FN-0741 (2003).

[3] J. Anderson et al., FERMILAB-Conf-06-470-E (2006).

[4] B. Casey et al., FERMILAB-Conf-06-469-E (2006).

[5] T. Edwards et al., FERMILAB-TM-2278-E (2004).

[6] E710 Collaboration, N.A. Amos et al., Phys. Lett. B301, 313 (1993).

[7] CDF Collaboration, F. Abe et al., Phys. Rev. D50, 5535 (1994).

[8] CDF Collaboration, T. Affolder et al., Phys. Rev. Lett. 87, 141802 (2001). 